\begin{tabular}{|c|c|}
\hline \multirow{3}{*}{ 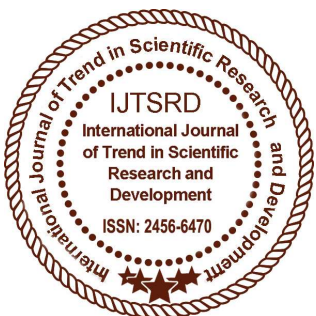 } & $\begin{array}{l}\text { International Journal of Trend in Scientific } \\
\text { Research and Development (IJTSRD) }\end{array}$ \\
\hline & International Open Access Journal \\
\hline & ISSN No: 2456 - 6470 | www.ijtsrd.com | Volume - 1 | Issue - 5 \\
\hline
\end{tabular}

\title{
Cell Cycle Dependent Regulation of Microtubule Dynamics by Microtubules Associated Proteins (MAPs) and its Misregulation causes Aneuploidy
}

\author{
Aijaz Rashid* \\ Postdoctoral Research Fellow, \\ National Institutes of Health, NIH, USA \\ *Corresponding author: clbaijaz@gmail.com
}

\author{
Shazia Ahad \\ Indian Institute of Technology(IIT) Bombay, \\ Maharastra, India
}

\begin{abstract}
Microtubules are intrinsic dynamic polymers but programmed regulation of microtubule dynamics through different phases of cell cycle is modulated by numerous proteins known as microtubules associated proteins (MAPs) and mitotic kinases. Proper attachment of microtubules with kinetochore and adequate tension generation leads to chromosomal congression at metaphase plate, that is followed by movement of sister chromatids towards opposite poles of cells. Impairment, in this process activates spindle assembly checkpoint proteins that hamper cell cycle progression and thus maintains genetic stability. Thus, regulation of microtubule dynamics is important in normal cell cycle progression and to prevent aneuploidy.
\end{abstract}

Although microtubules are intrinsic dynamic polymers but programmed regulation of microtubule dynamics through different phases of cell cycle is modulated by numerous proteins known as microtubules associated proteins (MAPs) and mitotic kinases[1-3]. Various MAPs present inside the cell maintain balance between polymeric and soluble pool of tubulin and are also responsible for reorientation of microtubule cytoskeleton. Microtubules perform indispensable role in mitosis. During mitosis microtubules attain high dynamic state, 20-100 folds increase in microtubule dynamics is observed during mitosis. The nucleation rate of microtubules from centrosomes during mitosis shows 7 -fold enhancement, compared to the interphase microtubules[4, 5]. There is drastic reduction in halflife of polymerized tubulin from 10 minute in interphase cell to merely $10-30 \mathrm{~s}$ in mitotic cell $[5,6]$.

The functional significance of enhanced dynamics after breakdown of nuclear envelope (prophase and prometaphase) is strategic kinetochore capture by highly dynamic microtubules. Highly dynamic microtubules display extended search for kinetochore and get hold of it by increasing in length followed by instantaneous shortening to bring it to metaphase plate[7].

This stochastic search-and-capture process displayed by microtubules emanating from one spindle pole capture kinetochore and a similar process allows other kinetochore of the same chromosomes to be captured by microtubules from other spindle pole, resulting in amphitelic kinetochore-microtubule attachments (51).This process involves proper tension generation and chromosomal congression at so called metaphase plate[8], followed by movement of sister chromotids towards opposite poles of cell. The poleward movement of chromatids is thought to be driven by shortening of kinetochore-attached microtubules. Some motor proteins also contribute in poleward movement of chromatids as well as in chromosomal congression process [9]. The segregation of chromosomes is followed by de-condensation in last phase of mitosis (telophase), thus two identical nuclei are formed. Finally cytokinesis takes place where in 
cytoplasm is divided by the contractile action of actin- $\quad$ position of cell[10]. myosin ring structures originating at the central

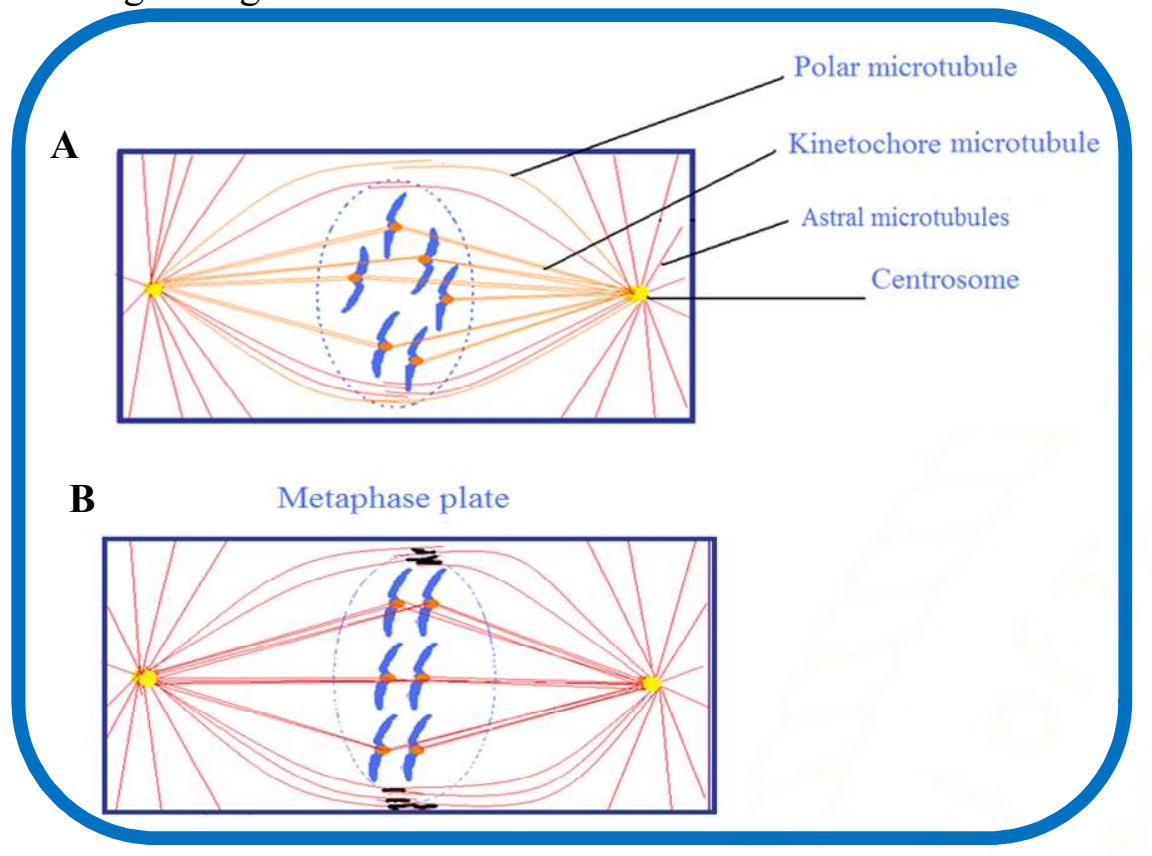

Figure 1: Microtubule dynamics and kinetochore capture: After the breakdown of nuclear envelope, microtubules emanating from centrosome called as kinetochore microtubules get hold of kinetochores of chromosomes and align chromosomes at medial position of cell called as metaphasic plate. Microtubule dynamics plays a vital role in kinetochore capture process. Panel A represents a cell in prometaphase and panel $\mathrm{B}$ represents cell in metaphase after chromosomes are aligned at metaphase plate.

During the progression of cell cycle, attachment of the microtubules to the chromosomes at kinetochores and their metaphasic alignment is of utmost importance because the mitotic cell has to ensure that chromosomes are rightly aligned in metaphase before the anaphase is started to ensure proper segregation of chromosomes to the newly formed daughter cells [11]. However, the failure in this process can lead to aberrant chromosome segregation with unaligned chromosomes which could be a cause for chromosomal instability and aneuploidy[12, 13]. To prevent aneuploidy, a stringent signal transduction pathway operates called as "the spindle assembly checkpoint". It is a cell cycle surveillance mechanism which postpones the onset of anaphase till all kinetochores are firmly adhered to spindle microtubules and proper tension is achieved[12]. The spindle checkpoint signaling mechanism comprises of several highly conserved proteins like Mad1, Mad2, Mad3/BubR1, Bub1, Bub3 and Mps1 [13]. During nuclear envelope breakdown kinetochore attachment process starts and spindle checkpoint proteins are activated and recruited to unattached kinetochores and kinetochores devoid of proper tension, resulting in the inhibition of anaphase-promoting-complex/cyclosome $(\mathrm{APC} / \mathrm{C})$. An activated spindle checkpoint prevents the beginning of anaphase through inhibition of protein proteolysis and hence prevention of chromatid separation[11, 13]. However, compromised spindle checkpoint mechanism may result in faulty separation of sister chromatids even in the presence of misaligned chromosomes that could be a cause for chromosomal instability (CIN) and hence result in gain or loss of chromosomes called as aneuploidy, a striking feature in human cancer[14]. Significantly, many tumors are known with weakened spindle checkpoint function, thus lack of sustenance of signal for repair of errors[15, 16]. Hence, an impaired spindle checkpoint may directly lead to chromosomal instability and tumorigenesis in human cancer[15]. 


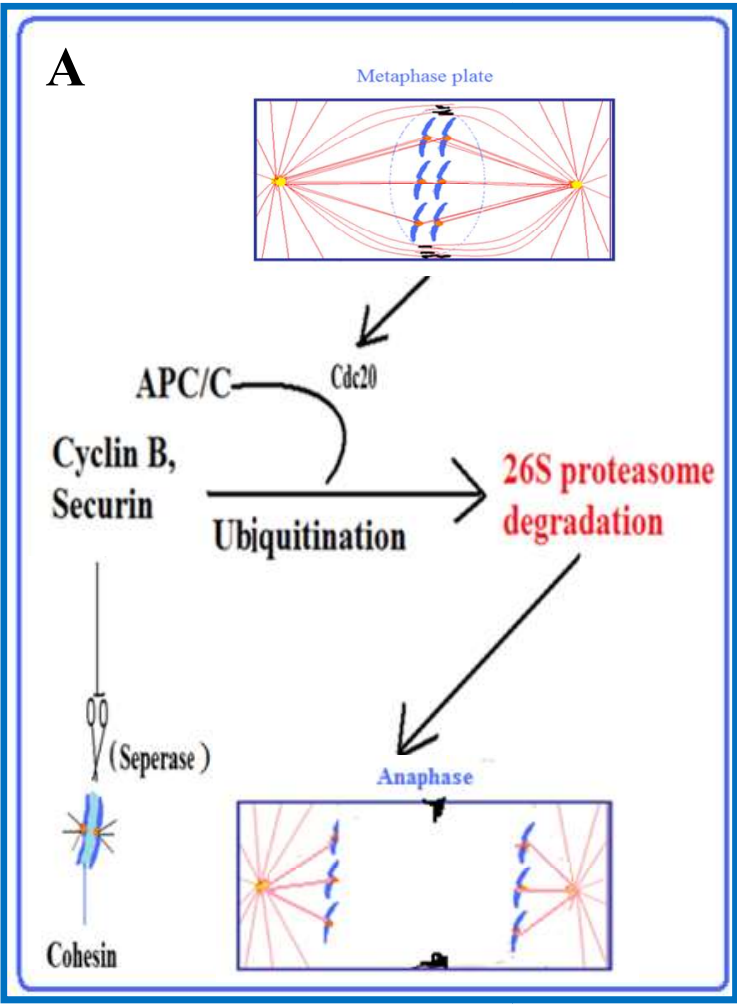

Figure 2: Mitotic progression: (A) On each kinetochore, when microtubules are adhered and proper tension is generated, the checkpoint proteins are satisfied and released. Then anaphase promoting complex $(\mathrm{APC} / \mathrm{C})$ promotes ubquitination of cyclin B and its degradation by $26 \mathrm{~S}$ proteasome thus providing the biochemical signal for cells to proceed towards anaphase. (B) On the other hand, unattached kinetochore and imbalanced kinetochore tension leads to recruitment of SAC proteins (Mad2, BubR1) to Kinetochore. SAC proteins sequester Cdc20 a cofactor of $\mathrm{APC} / \mathrm{C}$, thus inhibiting proteosomal degradation of Cyclin B and Securin. Cyclin B is a protein which regulates early phase of mitosis and securin is inhibitor of separase. Thus, prevention of proteosomal degradation of Cyclin B and Securin leads to mitotic block.

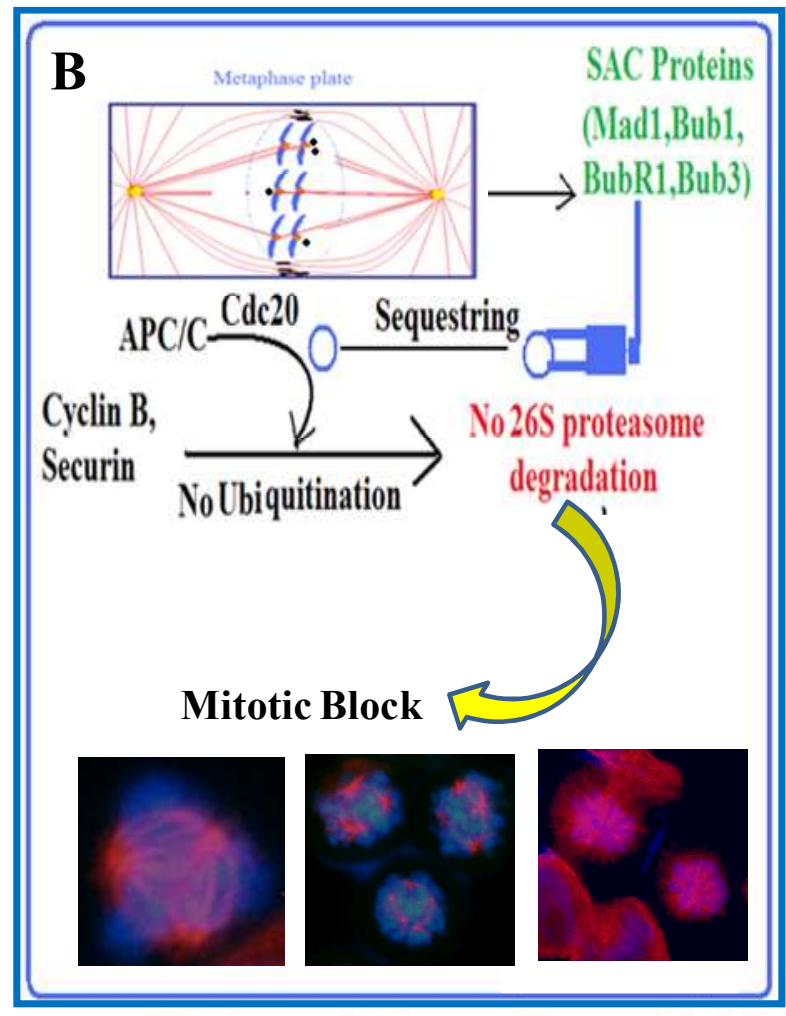

\section{REFERENCES:}

1) Walczak, C.E., Microtubule dynamics and tubulin interacting proteins. Curr Opin Cell Biol. 2000 Feb;12(1):52-6., 2000.

2) Maccioni, R.B. and V. Cambiazo, Role of microtubule-associated proteins in the control of microtubule assembly. Physiol Rev. 1995 Oct;75(4):835-64., 1995.

3) Vatti, A., et al., Original antigenic sin: $A$ comprehensive review. J Autoimmun, 2017. 83: p. 12-21.

4) Schmidt, M. and H. Bastians, Mitotic drug targets and the development of novel anti-mitotic anticancer drugs. Drug Resist Updat. 2007 AugOct;10(4-5):162-81. Epub 2007 Jul 31., 2007.

5) Piehl, M., et al., Centrosome maturation: measurement of microtubule nucleation throughout the cell cycle by using GFP-tagged EB1. Proc Natl Acad Sci U S A. 2004 Feb 10;101(6):1584-8. Epub 2004 Jan 27., 2004.

6) Saxton, W.M., et al., Tubulin dynamics in cultured mammalian cells. J Cell Biol. 1984 Dec;99(6):2175-86., 1984.

7) Hayden, J.H., S.S. Bowser, and C.L. Rieder, Kinetochores capture astral microtubules during 
chromosome attachment to the mitotic spindle: direct visualization in live newt lung cells. J Cell Biol. 1990 Sep;111(3):1039-45., 1990.

8) Cleveland, D.W., Y. Mao, and K.F. Sullivan, Centromeres and kinetochores: from epigenetics to mitotic checkpoint signaling. Cell. 2003 Feb 21;112(4):407-21., 2003.

9) Maiato, H., P. Sampaio, and C.E. Sunkel, Microtubule-associated proteins and their essential roles during mitosis. Int Rev Cytol. 2004;241:53-153., 2004.

10) Fededa, J.P. and D.W. Gerlich, Molecular control of animal cell cytokinesis. Nat Cell Biol. 2012 May 2;14(5):440-7. doi: 10.1038/ncb2482., 2012.

11) Millband, D.N., L. Campbell, and K.G. Hardwick, The awesome power of multiple model systems: interpreting the complex nature of spindle checkpoint signaling. Trends Cell Biol. 2002 May;12(5):205-9., 2002.
12) Hardwick, K.G., et al., Activation of the budding yeast spindle assembly checkpoint without mitotic spindle disruption. Science. 1996 Aug 16;273(5277):953-6., 1996.

13) Bharadwaj, R. and $\mathrm{H}$. Yu, The spindle checkpoint, aneuploidy, and cancer. Oncogene. 2004 Mar 15;23(11):2016-27., 2004.

14) Jallepalli, P.V. and C. Lengauer, Chromosome segregation and cancer: cutting through the mystery. Nat Rev Cancer. 2001 Nov;1(2):109-17., 2001.

15) Wassmann, K. and R. Benezra, Mitotic checkpoints: from yeast to cancer. Curr Opin Genet Dev. 2001 Feb;11(1):83-90., 2001.

16) Weaver, B.A. and D.W. Cleveland, Does aneuploidy cause cancer? Curr Opin Cell Biol. 2006 Dec;18(6):658-67. Epub 2006 Oct 12., 2006. 